\title{
Die Instrumente der Zivilisation
}

\section{Samia Hurst}

Prof. Dr. med., Mitglied der Redaktion

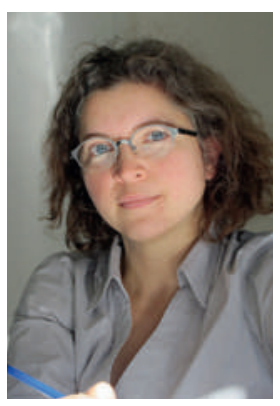

In dieser Ausgabe geben uns Bodenmann et al. eine gute Beschreibung der waadtländischen Massnahmen, mit deren Hilfe den gesundheitsspezifischen Bedürfnissen der Asylsuchenden begegnet werden soll [1]. Angesichts der - auch in der Schweiz - steigenden Anzahl von Zwangsmigranten ist es wichtig, dass wir unsere Strukturen entsprechend anpassen und Pflegeleistungen anbieten, die diesen Namen verdienen, ohne dabei unsere Gesundheitsdienste zu überlasten. Vor diesem Hintergrund bieten konkrete Beispiele Hilfestellung für jene Dienste, die diesen Schritt noch nicht gegangen sind.

Die Ereignisse zwischen dem Entscheid, diesen Artikel veröffentlichen zu wollen, und dem Zeitpunkt des eigentlichen Niederschreibens dieser Zeilen machen eine Bezugnahme auf die Attentate von Paris natürlich unumgänglich. Bereits vor den Attentaten gestaltete sich die korrekte Aufnahme und ordentliche Behandlung der aus Syrien geflohenen Menschen nicht einfach, und nun scheint es noch schwieriger zu werden. Die Positionen verhärten sich, Aufnahmeversprechen werden zurückgenommen, die Angst vor Terroristen, die unsere Länder unterwandern könnten, mischt sich noch deutlicher mit der Besorgnis, den Zustrom von Flüchtlingen nicht beherrschen zu können.

\section{Wer je den islamistischen Terror aus der Nähe} erlebt hat, dürfte kaum daran interessiert sein, in Europa einen Gottesstaat errichten zu wollen.

Diese Ängste sind rational. Jedenfalls sind sie nicht unvernünftig. Das von den gegenwärtig in unserem Land Ankommenden gezeigte «Bild des Elends» zeugt von der Durchquerung von Kriegs- und Notstandsgebieten, in denen die Instrumente der Zivilisation nur noch teilweise oder überhaupt nicht mehr wirksam sind [2]. Ohne Wasser und Nahrung, ohne Schutz gegen Kälte und Aggression, ohne Identitätskontrolle gilt es diese Reise zu ertragen, anscheinend ohne auf die geringste Unterstützung einer funktionierenden menschlichen Gemeinschaft hoffen zu können. Das ist in vielerlei Hinsicht gefährlich. Ja, diese Menschen haben dringende Bedürfnisse. Mit dem Einbruch des Winters werden wir uns darauf gefasst machen müssen, neben den Toten im Meer auch Menschen zu beklagen, die nach ihrer Ankunft auf europäischem Boden erfrieren. Ja, diese Menschen kommen in grosser Zahl und übersteigen die normalen Kapazitäten der Sicherheitskontrollen. Es ist richtig, dass Menschen, die den islamistischen Terror im Nahen Osten aus der Nähe erlebt haben, wohl kaum daran interessiert sein dürften, in Europa einen Gottesstaat errichten zu wollen [3]. Es ist auch richtig, dass ohne Kontrollen nicht garantiert werden kann, dass sich nicht doch Selbstmordattentäter unter ihnen befinden, auch wenn es keine Beispiele dafür gibt.

Vor diesem Hintergrund erscheinen wieder ganz schnell die klassischen Demarkationslinien zwischen jenen, die im Namen der menschlichen Solidarität nach Öffnung rufen, und jenen, die im Namen der Sicherheit Ab-

\section{Wir sollten nicht verzagen, sondern intelligente} Lösungen finden.

schottung verlangen. Wenn wir zwischen diesen Werten entscheiden, als würde es sich um rivalisierende Optionen handeln, vergessen wir dabei, dass beide zu unserer Gesellschaft gehören. Vielleicht vergessen wir dabei auch, zu welchen Leistungen die Instrumente unserer Zivilisation fähig sind. Flüchtlinge gut unterzubringen, ihre Sicherheit und die unserer Bevölkerung zu gewährleisten - all dies ist eine grosse Herausforderung, zu deren Bewältigung wir aber in der Lage sein sollten. Die Gesundheitsdienste bereiten sich vor. Andere auch. Rufe werden vernehmbar, die Dinge vor Ort lösen zu wollen, es den Asylsuchenden zu ermöglichen, legal ein Flugzeug zu besteigen, anstatt ihr Leben auf der Flucht zu riskieren. Wir sollten nicht verzagen, sondern intelligente Lösungen finden. «Aber wie sollen wir bei sehr schwierigen Problemen vorgehen?», fragen uns gelegentlich Studierende und junge Ärzte in den Seminaren. Unsere Antwort: das Erlernte umsetzen, aber noch besser als sonst!

\section{Literatur}

1 Bodenmann P, Ninane F, Pahud-Vermeulen B, Dory E, Monnat M, Cornuz J, et al. Afflux des migrants. Schweiz Ärztezeitung 2015;96(50/51):1881-3.

2 Gulland A. The refugee crisis: what care is needed and how can doctors help? BMJ. 2015;351:h4881. PubMed PMID: 26359442

3 Jula R. Ich bin ein Syrer. NZZ am Sonntag. 2015 5.11.2015. 